\title{
BUBBLE TREE FOR APPROXIMATE HARMONIC MAPS
}

\author{
XIANGRONG ZHU
}

(Communicated by Michael Wolf)

AbSTRACT. In this paper, we set up the complete bubble tree theory for approximate harmonic maps from a Riemann surface with tension fields bounded in Zygmund class $L \ln ^{+} L$. Some special cases of this theory have previously been used in a number of papers.

On the other hand, one can see that this bubble tree theory is not true for the general target manifold if we only assume that the tension fields are bounded in $L^{1}$ uniformly.

\section{INTRODUCTION AND MAIN RESULTS}

Let $(M, g)$ be a closed Riemannian manifold and $(N, h)$ be a Riemannian manifold without boundary. For a map $u$ from $M$ to $N$ in $W^{1,2}(M, N)$, the energy density of $u$ is defined by

$$
e(u)=\frac{1}{2}|d u|^{2}=\operatorname{Trace}_{g} u^{*} h,
$$

where $u^{*} h$ is the pull-back of the metric tensor $h$.

The energy of the map $u$ is defined as

$$
E(u)=\int_{M} e(u) d V
$$

where $d V$ is the volume element of $(M, g)$.

A map $u \in C^{1}(M, N)$ is called a harmonic map if it is a critical point of the energy $E$.

By the Nash embedding theorem we know that $(N, h)$ can be isometrically embedded into a Euclidean space $R^{K}$ with some positive integer $K$. Then $(N, h)$ may be considered as a submanifold of $R^{K}$ with the metric induced from the Euclidean metric. Thus a map $u \in C^{1}(M, N)$ can be considered as a map of $C^{1}\left(M, R^{K}\right)$ whose image lies on $N$. In this sense we can get the following Euler-Lagrange equation:

$$
\triangle u=A(u)(d u, d u) \text {. }
$$

The tension field $\tau(u)$ is defined by

$$
\tau(u)=\triangle_{M} u-A(u)(d u, d u),
$$

where $A(u)(d u, d u)$ is the second fundamental form of $N$ in $R^{K}$. So $u$ is harmonic means that $\tau(u)=0$.

The harmonic maps are of special interest when $M$ is a Riemann surface, as the energy is conformal invariant in this case.

Received by the editors August 2, 2011 and, in revised form, July 14, 2012.

2010 Mathematics Subject Classification. Primary 53C43, 58E20.

The author was supported by NSFC(11101372). 
Consider a sequence of maps $u_{n}$ from Riemann surface $M$ to $N$ with bounded energies. It is clear that $u_{n}$ converges weakly to $u$ in $W^{1,2}(M, N)$ for some $u \in$ $W^{1,2}(M, N)$. But in general, it may not converge to $u$ strongly in $W^{1,2}(M, N)$.

The space $L \ln ^{+} L$ (Zygmund class) is defined by

$$
\left\{f:\|f\|_{L \ln ^{+} L}=\int|f(x)| \ln (2+|f(x)|) d x<\infty\right\} .
$$

Note that $\|\cdot\|_{L \ln ^{+} L}$ isn't a standard norm, but we can find a norm in the space $L \ln ^{+} L$

We may use the following quantity in the space $L \ln ^{+} L$ :

$$
\|f\|_{L \ln ^{+} L}^{*}=\int_{0}^{\infty} f^{*}(t) \ln \left(2+\frac{1}{t}\right) d t,
$$

where $f^{*}(t)=\inf \{s \geq 0:|\{x:|f(x)|>s\}| \leq t\}$ is the nonincreasing rearrangement function of $f$.

We know that $\|\cdot\|_{L \ln { }^{+}}^{*}$ and $\|\cdot\|_{L \ln { }^{+}{ }_{L}}$ can be controlled by each other through suitable functions.

We will show how $u_{n}$ tends to $u$ weakly in $W^{1,2}(M, N)$. Our main result is the following bubble tree theorem. Without loss of generality, let $M$ be the unit disk $B_{1}$.

Theorem 1. Let $\left\{u_{n}\right\}$ be a sequence of maps from $B_{1}$ to $N$ in $W^{1,2}\left(B_{1}, N\right)$ with tension field $\tau\left(u_{n}\right)$. If $u_{n}$ tends to $u$ strongly in $W^{1,2}\left(B_{1} \backslash B_{\frac{1}{2}}, N\right)$, weakly in $W^{1,2}\left(B_{1}, N\right)$ and

$$
\left\|u_{n}\right\|_{W^{1,2}\left(B_{1}\right)}+\left\|\tau\left(u_{n}\right)\right\|_{L \ln ^{+}\left(B_{1}\right)} \leq \Lambda,
$$

then there exist a subsequence of $\left\{u_{n}\right\}$ (we still denote it by $\left\{u_{n}\right\}$ ) and some nonnegative integer $m$. For any $i=1, \ldots, m$, there exist points $x_{n}^{i}$, positive numbers $r_{n}^{i}$ and nonconstant harmonic spheres $\psi_{i}$ (which we view as a map from $R^{2} \cup\{\infty\} \rightarrow N$ ) such that

(1) $x_{n}^{i} \rightarrow x^{i} \in B_{\frac{1}{2}}, r_{n}^{i} \rightarrow 0$ as $n \rightarrow \infty$.

(2) $\lim _{n \rightarrow \infty}\left(\frac{r_{n}^{i}}{r_{n}^{j}}+\frac{r_{n}^{j}}{r_{n}^{i}}+\frac{\left|x_{n}^{i}-x_{n}^{j}\right|}{r_{n}^{i}+r_{n}^{j}}\right)=\infty$ for any $i \neq j$.

(3) $\psi_{i}$ is the weak limit or strong limit of $u_{n}\left(x_{n}^{i}+r_{n}^{i} x\right)$ in $W_{L o c}^{1,2}\left(R^{2}, N\right)$. Moreover, if

$$
\lim _{n \rightarrow \infty} \frac{\left|x_{n}^{i}-x_{n}^{j}\right|}{r_{n}^{i}}=\infty, \forall j \neq i,
$$

then $u_{n}\left(x_{n}^{i}+r_{n}^{i} x\right) \rightarrow \psi_{i}$ strongly in $W_{L o c}^{1,2}\left(R^{2}, N\right)$.

(4) For any $x_{n} \rightarrow x_{0} \in B_{1}, r_{n} \rightarrow 0$, if for any $i=1,2, \ldots, m$, we have

$$
\lim _{n \rightarrow \infty}\left(\frac{r_{n}^{i}}{r_{n}}+\frac{r_{n}}{r_{n}^{i}}+\frac{\left|x_{n}^{i}-x_{n}\right|}{r_{n}^{i}+r_{n}}\right)=\infty,
$$

then $u_{n}\left(x_{n}+r_{n} x\right) \rightarrow 0$ weakly in $W_{L o c}^{1,2}\left(R^{2}, N\right)$.

Remark. Property (2) means that the sequences $u_{n}\left(x_{n}^{i}+r_{n}^{i} x\right)$ and $u_{n}\left(x_{n}^{j}+r_{n}^{j} x\right)$ do not tend to the same bubble weakly in $W_{L o c}^{1,2}\left(R^{2}, N\right)$. Property (3) shows the picture about the bubbles. Property (4) implies that there are no other bubbles for this subsequence. 
If there is no energy on the neck domain during blowing up, i.e.

$$
\lim _{\delta \rightarrow 0} \lim _{R \rightarrow \infty} \lim _{n \rightarrow \infty} E\left(u_{n}, \bigcup_{i}^{m} B\left(x^{i}, \delta\right) \backslash \bigcup_{i}^{m} B\left(x_{n}^{i}, r_{n}^{i} R\right)\right)=0,
$$

which is equivalent to

$$
\lim _{n \rightarrow \infty} E\left(u_{n}\right)=E(u)+\sum_{i=1}^{m} E\left(\psi_{i}\right),
$$

we say that the energy identity holds.

If there is no oscillation on the neck domain, i.e.

$$
\lim _{\delta \rightarrow 0} \lim _{R \rightarrow \infty} \lim _{n \rightarrow \infty} \sup _{x, y \in \bigcup_{i}^{m} B\left(x^{i}, \delta\right) \backslash \bigcup_{i}^{m} B\left(x_{n}^{i}, r_{n}^{i} R\right)}\left|u_{n}(x)-u_{n}(y)\right|=0,
$$

we say that there is no neck or necklessness during blowing up. The geometrical meaning is that the image $u\left(B_{1}\right) \cup \bigcup_{i=1}^{m} \psi_{j}\left(R^{2}\right)$ is a connected set if there is no neck during blowing up.

When $\tau\left(u_{n}\right)=0$ or $\tau\left(u_{n}\right)$ are bounded in $L^{2}$, the bubble tree, energy identity and necklessness have been proved in [1,4-8]. Though we have not found the complete proof of bubble tree theorem, it seems that it is not difficult to extend the bubble tree theorem to the case where $\tau\left(u_{n}\right)$ are bounded in $L^{p}$ for some $p>1$. In 3 we extended the energy identity and necklessness for maps with tension fields bounded in $L^{\frac{6}{5}}$.

In [2], when the target manifold is a standard sphere, we proved the bubble tree theorem and the energy identity for a sequence of $u_{n}$ with bounded energies and tension fields bounded in $L \ln ^{+} L$. It is worth pointing out that there may exist a positive neck in this case.

Our proof of the bubble tree theorem in 2] depends on a convergence result and a new compactness theorem. The convergence result was proved via a div-curl lemma and it is not valid for the general target manifold.

Here we use a special induction on the quantity $\sup _{n} E\left(u_{n}, B_{1}\right)$ to prove the main theorem. Also our proof depends on the compactness theorem for approximate harmonic maps proved in [2] and a convergence lemma for the general target manifold.

Throughout this paper, without illustration the letter $C$ denotes a positive constant which may depend on $\Lambda, N$ and may vary in different cases. Furthermore we do not distinguish the sequence and its subsequence.

\section{Proof OF THE MAIN THEOREM}

We recall the following compactness theorem ([2], Theorem 1.2).

Theorem 2. Let $N$ be a compact Riemannian manifold. Assume that a sequence of maps $u_{n} \in W^{1,2}\left(B_{1}, N\right)$ satisfies

$$
\left\|u_{n}\right\|_{W^{1,2}\left(B_{1}\right)}+\left\|\tau\left(u_{n}\right)\right\|_{L \ln ^{+}\left(B_{1}\right)} \leq \Lambda .
$$

There exists a positive constant $\epsilon_{N}$ which depends only on the target manifold such that if $E\left(u_{n}, B_{1}\right) \leq \epsilon_{N}$, then there exists a subsequence of $u_{n}$ (still denoted by $u_{n}$ ) and $u$ such that

$$
\lim _{n \rightarrow \infty}\left\|u_{n}-u\right\|_{W^{1,2}\left(B_{\frac{1}{2}}\right)}=0 .
$$


It is easy to see that this theorem is not true if we only assume that $\tau\left(u_{n}\right)$ are bounded in $L^{1}$.

The point $x \in B_{1}$ is called a concentration point of $u_{n}$ if for any $r, 0<r<1-|x|$,

$$
\sup _{n} E\left(u_{n}, B(x, r)\right)>\epsilon_{N} .
$$

By Theorem 2, if $x_{0}$ is not a concentration point of $u_{n}$, then $u_{n}$ has a subsequence converging strongly in $W^{1,2}$-norm near $x_{0}$.

We show that there exists a subsequence of $u_{n}$ (we still denote it by $u_{n}$ ) that has only finite concentration points. If it is not true, take a positive integer $J>\frac{\Lambda}{\epsilon_{N}}$. Then we can find a subsequence of $u_{n}$ that has at least $J$ concentration points $x_{1}, x_{2}, \ldots, x_{J}$. So we have

$$
\lim _{n \rightarrow \infty} E\left(u_{n}, B_{1}\right) \geq \sum_{1}^{J} \lim _{r \rightarrow 0} \lim _{n \rightarrow \infty} E\left(u_{n}, B\left(x_{i}, r\right)\right)>J \epsilon_{N}>\Lambda,
$$

which contradicts the assumption.

As a corollary, we get that immediately

Lemma 3. Suppose $M$ is a closed Riemannian surface without boundary. Then for any nontrivial harmonic map $u$ from $M$ to $N$, there holds

$$
E(u) \geq \epsilon_{N}
$$

We also need a convergence lemma.

Lemma 4. Suppose that $u_{n}$ are maps from a Riemann surface $M$ to $N$ with energies bounded and tension fields $\tau\left(u_{n}\right)$ bounded in $L \ln ^{+} L$, i.e.

$$
\left\|u_{n}\right\|_{W^{1,2}(M)}+\left\|\tau\left(u_{n}\right)\right\|_{L \ln ^{+} L(M)} \leq \Lambda \text {. }
$$

Then there exists $u \in W^{1,2}(M, N)$ and a subsequence of $u_{n}$ (still denoted by $u_{n}$ ) such that $u_{n} \rightarrow u$ weakly in $W^{1,2}(M, N)$ and

$$
\triangle u_{n}+A\left(u_{n}\right)\left(d u_{n}, d u_{n}\right) \rightarrow \triangle u+A(u)(d u, d u) \text { in } L^{1}(M) .
$$

Remark. When the target manifold is a standard sphere or the Euclidean space, this lemma is true without any conditions on $\tau\left(u_{n}\right)$.

Proof. Passing to a subsequence, it is easy to assume that $u_{n} \rightarrow u$ weakly in $W^{1,2}$ and $u_{n} \rightarrow u$ in $L^{1}$.

Without loss of generality suppose that there is only one concentration point $\{0\} \in M$ for the sequence $u_{n}$; in this case

$$
\left|\nabla u_{n}\right|^{2} \rightarrow|\nabla u|^{2}+a \delta_{0}
$$

where $a>0$. By Theorem 2 we know that $u_{n} \rightarrow u$ strongly in $W^{1,2}\left(M \backslash B_{\delta}\right)$ for any $\delta>0$.

For any positive integer $k$, set $F_{n, k}=\left\{x \in M:\left|u_{n}(x)-u(x)\right|>\frac{1}{k}\right\}$. Obviously there holds $\left|F_{n, k}\right| \rightarrow 0$ as $n \rightarrow \infty$. So for any $k$, there exists $n_{k}$ such that $\left|F_{n_{k}, k}\right|<$ $2^{-k}$. Choose the subsequence $u_{n_{k}}$ and rewrite it as $u_{n}$. Then for any $n$ we have

$$
\left|\left\{x \in M:\left|u_{n}(x)-u(x)\right|>\frac{1}{n}\right\}\right|<2^{-n} .
$$

Take $n_{\delta}$ such that $2^{-n_{\delta}}<\delta$ and set $F_{0}=\bigcup_{n>n_{\delta}}\left\{x \in M:\left|u_{n}(x)-u(x)\right|>\frac{1}{n}\right\}$. Then $\left|F_{0}\right|<2^{-n_{0}}<\delta$ and $u_{n} \rightarrow u$ strongly in $L^{\infty}\left(M \backslash F_{0}\right)$. 
Now we show that $A\left(u_{n}\right)\left(d u_{n}, d u_{n}\right) \rightarrow A(u)(d u, d u)$ in $L^{1}\left(M \backslash\left(F_{0} \cup B_{\delta}\right)\right)$. As the coefficient of the second fundamental form is smooth in $N$, it can be shown that

$$
\begin{aligned}
& \left|A\left(u_{n}\right)\left(d u_{n}, d u_{n}\right)-A(u)(d u, d u)\right| \\
= & \mid\left(A\left(u_{n}\right)\left(d u_{n}, d u_{n}\right)-A\left(u_{n}\right)\left(d u_{n}, d u\right)\right)+\left(A\left(u_{n}\right)\left(d u_{n}, d u\right)-A\left(u_{n}\right)(d u, d u)\right) \\
& +\left(A\left(u_{n}\right)(d u, d u)-A(u)(d u, d u)\right) \mid \\
\leq & \left|A\left(u_{n}\right)\right|\left(\left|\nabla u_{n}\right|\left|\nabla u_{n}-\nabla u\right|+|\nabla u|\left|\nabla u_{n}-\nabla u\right|\right)+\left|A\left(u_{n}\right)-A(u)\right||\nabla u|^{2} \\
\leq & C\left(\left(\left|\nabla u_{n}\right|+|\nabla u|\right)\left|\nabla\left(u_{n}-u\right)\right|+\left|u_{n}-u\right||\nabla u|^{2}\right) .
\end{aligned}
$$

Because $u_{n} \rightarrow u$ in $L^{\infty}\left(M \backslash F_{0}\right)$ and $u_{n} \rightarrow u$ in $W^{1,2}\left(M \backslash B_{\delta}\right)$, we can get that

$$
\begin{aligned}
& \lim _{n \rightarrow \infty}\left\|A\left(u_{n}\right)\left(d u_{n}, d u_{n}\right)-A(u)(d u, d u)\right\|_{L^{1}\left(M \backslash\left(F_{0} \cup B_{\delta}\right)\right)} \\
\leq & C \lim _{n \rightarrow \infty}\left(\left\|\nabla\left(u_{n}+u\right)\right\|_{2}\left\|\nabla\left(u_{n}-u\right)\right\|_{L^{2}\left(M \backslash B_{\delta}\right)}+\left\|u_{n}-u\right\|_{L^{\infty}\left(M \backslash F_{0}\right)}\|\nabla u\|_{2}^{2}\right) \\
(1)= & 0 .
\end{aligned}
$$

As $\triangle u_{n}=-A\left(u_{n}\right)\left(d u_{n}, d u_{n}\right)+\tau\left(u_{n}\right)$ are bounded in $L^{1}$, we can assume that

$$
\left|\triangle u_{n}\right| \rightarrow f+b \delta_{0},
$$

where $b \geq 0$. It is worth pointing out that here $b$ may be equal to 0 . So there exists $f_{0} \in L^{1}\left(M \backslash B_{\delta}\right)$ such that $\triangle u_{n} \rightarrow f_{0}$ in $L^{1}\left(M \backslash B_{\delta}\right)$. On the other hand, as $\nabla u_{n} \rightarrow \nabla u$ weakly in $L^{2}(M)$, there holds $\triangle u_{n} \rightarrow \triangle u$ in the sense of distribution. So it must be $\triangle u=f_{0}$ on $M \backslash B_{\delta}$, i.e.

$$
\lim _{n \rightarrow \infty}\left\|\triangle u_{n}-\triangle u\right\|_{L^{1}\left(M \backslash B_{\delta}\right)}=0 .
$$

Take a cut-off function $\varphi \in C_{0}^{\infty}\left(B_{2}\right)$ with $\varphi=1$ on $B_{1}$ and $\varphi_{\delta}(x)=\varphi\left(\frac{x}{\delta}\right)$. It can be shown that

$$
\begin{aligned}
& \left(\triangle u_{n}+A\left(u_{n}\right)\left(d u_{n}, d u_{n}\right)\right)-(\triangle u+A(u)(d u, d u)) \\
= & \left(1-\varphi_{\delta}\right)\left(1-\chi_{F_{0}}\right)\left[\left(\Delta_{n}-\triangle u\right)+\left(A\left(u_{n}\right)\left(d u_{n}, d u_{n}\right)-A(u)(d u, d u)\right)\right] \\
& +\left(\varphi_{\delta}+\chi_{F_{0}}-\varphi_{\delta} \chi_{F_{0}}\right)\left(\triangle u_{n}+A\left(u_{n}\right)\left(d u_{n}, d u_{n}\right)\right) \\
& -\left(\varphi_{\delta}+\chi_{F_{0}}-\varphi_{\delta} \chi_{F_{0}}\right)(\triangle u+A(u)(d u, d u)) \\
= & -\left(1-\varphi_{\delta}\right) \chi_{F_{0}} \triangle u-\left(\varphi_{\delta}+\chi_{F_{0}}-\varphi_{\delta} \chi_{F_{0}}\right) A(u)(d u, d u) \\
& +\left(1-\varphi_{\delta}\right)\left(1-\chi_{F_{0}}\right)\left[\left(\triangle u_{n}-\triangle u\right)+\left(A\left(u_{n}\right)\left(d u_{n}, d u_{n}\right)-A(u)(d u, d u)\right)\right] \\
& +\left(\varphi_{\delta}+\chi_{F_{0}}-\varphi_{\delta} \chi_{F_{0}}\right) \tau\left(u_{n}\right) \\
& -\varphi_{\delta} \triangle u .
\end{aligned}
$$

At first, as $\triangle u \in L^{1}\left(M \backslash B_{\delta}\right)$ and $|\nabla u|^{2} \in L^{1}(M)$, by the absolute continuity, for any $\epsilon>0$, let $\delta$ be small enough such that

$$
\int_{M}\left(1-\varphi_{\delta}\right) \chi_{F_{0}}|\triangle u| d x+\int_{M}\left(\varphi_{\delta}+\chi_{F_{0}}-\varphi_{\delta} \chi_{F_{0}}\right)|A(u)(d u, d u)| d x<\epsilon .
$$

From (11) and (2), there exists $n_{\epsilon}>n_{\delta}$, such that for any $n>n_{\epsilon}$,

(4) $\int_{M}\left(1-\varphi_{\delta}\right)\left(1-\chi_{F_{0}}\right)\left|\left(\triangle u_{n}-\triangle u\right)+\left(A\left(u_{n}\right)\left(d u_{n}, d u_{n}\right)-A(u)(d u, d u)\right)\right| d x<\epsilon$. 
On the other hand, some simple computations show that

$$
\begin{aligned}
& \int_{M}\left(\varphi_{\delta}+\chi_{F_{0}}-\varphi_{\delta} \chi_{F_{0}}\right)\left|\tau\left(u_{n}\right)\right| d x \\
\leq & \int_{F_{0} \cup B_{2 \delta}}\left|\tau\left(u_{n}\right)\right| d x \\
\leq & \int_{0}^{\left|F_{0} \cup B_{2 \delta}\right|}\left|\tau\left(u_{n}\right)^{*}\right| d t \\
\leq & \int_{0}^{C \delta^{2}}\left(\left|\tau\left(u_{n}\right)^{*}\right| \ln \frac{1}{t}\right)\left(\ln \frac{1}{t}\right)^{-1} d t \\
\leq & C\left(\ln \frac{1}{\delta}\right)^{-1}\left\|\tau\left(u_{n}\right)\right\|_{L \ln }^{*} L \\
\leq & C\left(\ln \frac{1}{\delta}\right)^{-1} .
\end{aligned}
$$

At last, for any $\phi \in C^{\infty}(M, N)$, we have

$$
\begin{aligned}
& \left|\int_{M} \varphi_{\delta} \phi \cdot \Delta u d x\right| \\
= & \left|\int_{M} \nabla\left(\varphi_{\delta} \phi\right) \nabla u d x\right| \\
\leq & \left\|\nabla\left(\varphi_{\delta} \phi\right)\right\|_{2}\|\nabla u\|_{L^{2}\left(B_{2 \delta}\right)} \\
\leq & C\left(1+\delta\|\nabla \phi\|_{\infty}\right) \epsilon^{\frac{1}{2}} .
\end{aligned}
$$

Here we use the fact that $\int_{B_{2 \delta}}|\nabla u|^{2} d x<\epsilon$.

For any $\phi \in C^{\infty}(M, N)$ and $\epsilon>0$, take $\delta$ small enough. Then from (3), (4), (5) and (6) there exists a positive integer $n_{\epsilon}$ such that for any $n>n_{\epsilon}$ there holds

$$
\begin{aligned}
& \left|\int_{M}\left[\left(\triangle u_{n}+A\left(u_{n}\right)\left(d u_{n}, d u_{n}\right)\right)-(\triangle u+A(u)(d u, d u))\right] \phi d x\right| \\
< & C\left(\left(\ln \frac{1}{\delta}\right)^{-1}+\epsilon^{\frac{1}{2}}\right)\|\nabla \phi\|_{C^{1}} \\
< & C \epsilon^{\frac{1}{2}} .
\end{aligned}
$$

So we get that $\triangle u_{n}+A\left(u_{n}\right)\left(d u_{n}, d u_{n}\right) \rightarrow \triangle u+A(u)(d u, d u)$ in the sense of distribution, i.e. $\tau\left(u_{n}\right) \rightarrow \tau(u)$ in the sense of distribution. On the other hand, as $\tau\left(u_{n}\right)$ are bounded in $L^{1}$, there exists a subsequence of $\tau \in L^{1}$ and $\tau \in L^{1}$ such that

$$
\tau\left(u_{n}\right) \rightarrow \tau+\mu
$$

with $|\operatorname{spt}(\mu)|=0$. Because $\tau\left(u_{n}\right)$ are bounded in $L \ln ^{+} L$, there is no concentration point for the sequence $\tau\left(u_{n}\right)$, i.e. there must be $\mu=0$. So there must be $\tau(u)=\tau$ which implies that $\triangle u_{n}+A\left(u_{n}\right)\left(d u_{n}, d u_{n}\right) \rightarrow \triangle u+A(u)(d u, d u)$ in $L^{1}(M)$.

As a simple application of this lemma, we show that

Lemma 5. Suppose that $u_{n}$ are maps from $B_{1}$ to $N$ with bounded energies and tension fields $\tau\left(u_{n}\right)$ bounded in $L \ln ^{+} L$, i.e.

$$
\left\|u_{n}\right\|_{W^{1,2}\left(B_{1}\right)}+\left\|\tau\left(u_{n}\right)\right\|_{L \ln ^{+}\left(B_{1}\right)} \leq \Lambda .
$$

For any sequence $r_{n} \rightarrow 0$ and $x_{n} \in B_{\frac{1}{2}}$, there is a subsequence of $w_{n}(x)=$ $u_{n}\left(x_{n}+r_{n} x\right)$ which converges to a harmonic map $w$ weakly in $W_{L o c}^{1,2}\left(R^{2}, N\right)$. 
Some simple computations show that $\tau\left(w_{n}\right)$ are bounded in $L \ln ^{+} L$ and we have $\int_{B_{R}}\left|\tau\left(w_{n}\right)\right| d x \rightarrow 0$. We omit the details.

Proof of the main theorem. Now we use the induction on the quantity $I=$ $\lim _{n \rightarrow \infty} \frac{\left|E\left(u_{n}, B_{1}\right)-E\left(u, B_{1}\right)\right|}{\epsilon_{N}}$ to prove the main theorem.

Step 1. If $\lim _{n \rightarrow \infty} \frac{\left|E\left(u_{n}, B_{1}\right)-E\left(u, B_{1}\right)\right|}{\epsilon_{N}}<2$, it is easy to see that there is at most one bubble. In this case, if $E\left(u_{n}, B_{1}\right)<\frac{\epsilon_{N}}{2}$, take $r_{n}^{1}=1, x_{n}^{1}=0$. Otherwise by the continuity of integral, for any $n$, we can find $r_{n}^{1}, x_{n}^{1} \in B_{\frac{1}{2}}$ such that

$$
E\left(u_{n}, B\left(x_{n}^{1}, r_{n}^{1}\right)\right)=\sup _{B\left(y, r_{n}^{1}\right) \subset B_{1}} E\left(u_{n}, B\left(y, r_{n}^{1}\right)\right)=\frac{\epsilon_{N}}{2} .
$$

If $\lim _{n \rightarrow \infty} r_{n}^{1}>0$, there is no bubble, otherwise set $w_{n}^{1}(x)=u_{n}\left(x_{n}^{1}+r_{n}^{1} x\right)$. It is easy to check that

$$
E\left(w_{n}^{1}, B_{1}\right)=\sup _{y} E\left(w_{n}^{1}, B(y, 1)\right)=E\left(u_{n}, B\left(x_{n}^{1}, r_{n}^{1}\right)\right)=\frac{\epsilon_{N}}{2} .
$$

For any $R>0$, there holds $E\left(w_{n}^{1}, B_{R}\right) \leq \Lambda$, so by Theorem 2 there exists $\psi^{1} \in$ $W^{1,2}\left(R^{2}\right)$ such that $w_{n}^{1} \rightarrow \psi^{1}$ strongly in $W_{L o c}^{1,2}\left(R^{2}\right)$.

Obviously $\psi^{1}$ is not a constant map. From Lemma 5 we know $\psi^{1}$ is a weakly harmonic map. By Helein's theorem, $\psi^{1}$ is a nonconstant smooth harmonic map. Thus we prove the theorem in this case.

Step 2. Assume that when $I=\lim _{n \rightarrow \infty} \frac{\left|E\left(u_{n}, B_{1}\right)-E\left(u, B_{1}\right)\right|}{\epsilon_{N}}<k$, the theorem is true. Now suppose that

$$
I=\lim _{n \rightarrow \infty} \frac{\left|E\left(u_{n}, B_{1}\right)-E\left(u, B_{1}\right)\right|}{\epsilon_{N}}<k+1 .
$$

By the same argument in Step 1 we can show that there is no bubble or obtain the first bubble $\psi^{1}$ which is the strong limit of $w_{n}^{1}(x)=u_{n}\left(x_{n}^{1}+r_{n}^{1} x\right)$ in $W_{L o c}^{1,2}\left(R^{2}\right)$. It does not matter if we assume that $x_{n}^{1}=0$ for any $n$.

We claim that there exist $\epsilon>0$ and a sequence $t_{n} \rightarrow 0$ such that

(1) $\int_{B_{2 t_{n}} \backslash B_{t_{n}}}\left|\nabla u_{n}\right|^{2} d x>\epsilon, \forall n$;

(2) for any sequence $s_{n} \rightarrow 0$, if $\lim _{n \rightarrow \infty} \frac{s_{n}}{t_{n}}=\infty$, then there holds

$$
\lim _{n \rightarrow \infty} \int_{B_{2 s_{n}} \backslash B_{s_{n}}}\left|\nabla u_{n}\right|^{2} d x=0 .
$$

If it is not true, then we can get infinity sequences $t_{n}^{j} \rightarrow 0$ such that $\lim _{n \rightarrow \infty} \frac{t_{n}^{j+1}}{t_{n}^{j}}=$ $\infty$ and

$$
\lim _{n \rightarrow \infty} \int_{B_{2 t_{n}^{j} \backslash B_{t_{n}^{j}}}}\left|\nabla u_{n}\right|^{2} d x>0 .
$$

Set $v_{n}^{j}(x)=u_{n}\left(t_{n}^{j} x\right)$ and suppose that $v_{n}^{j} \rightarrow v^{j}$ weakly in $W_{L o c}^{1,2}\left(R^{2}\right)$.

Case 1. If there exists a concentration point $x^{j} \in B_{4} \backslash B_{\frac{1}{2}}$ for the sequence $v_{n}^{j}$, then from Theorem 2 we have

$$
\begin{aligned}
\lim _{R \rightarrow \infty} \lim _{n \rightarrow \infty} E\left(v_{n}^{j}, B_{R}\right) & \geq \lim _{n \rightarrow \infty} E\left(v_{n}^{j}, B_{4} \backslash B_{\frac{1}{2}}\right)+\lim _{n \rightarrow \infty} E\left(v_{n}^{j}, B_{\frac{1}{2}}\right) \\
& >\epsilon_{N}+\lim _{R \rightarrow \infty} \lim _{n \rightarrow \infty} E\left(v_{n}^{j-1}, B_{R}\right) .
\end{aligned}
$$


Case 2. If there is no concentration point on $B_{4} \backslash B_{\frac{1}{2}}$ for the sequence $v_{n}^{j}$, then from Theorem 2 and Lemma 5 we know that $v^{j}$ is a nonconstant harmonic map and the origin 0 is a concentration point for the sequence $v_{n}^{j}$. So there holds

$$
\begin{aligned}
\lim _{R \rightarrow \infty} \lim _{n \rightarrow \infty} E\left(v_{n}^{j}, B_{R}\right) & \geq E\left(v^{j}\right)+\lim _{\delta \rightarrow 0} \lim _{n \rightarrow \infty} E\left(v_{n}^{j}, B_{\delta}\right) \\
& >\epsilon_{N}+\lim _{R \rightarrow \infty} \lim _{n \rightarrow \infty} E\left(v_{n}^{j-1}, B_{R}\right) .
\end{aligned}
$$

So we always have

$$
\begin{aligned}
\lim _{n \rightarrow \infty}\left(E\left(u_{n}, B_{1}\right)-E(u)\right) & \geq \lim _{R \rightarrow \infty} \lim _{n \rightarrow \infty} E\left(v_{n}^{j}, B_{R}\right) \\
& >\epsilon_{N}+\lim _{R \rightarrow \infty} \lim _{n \rightarrow \infty} E\left(v_{n}^{j-1}, B_{R}\right) \\
& >j \epsilon_{N} .
\end{aligned}
$$

Take $j>k+1$; it is a contradiction, so the claim is true.

Set $\tilde{u}_{n}(x)=u_{n}\left(t_{n} x\right)$. Assume that $\tilde{u}_{n} \rightarrow \tilde{u}$ weakly in $W_{L o c}^{1,2}\left(R^{2}\right)$.

Case 1. Assume there exists a concentration point on $B_{4} \backslash B_{\frac{1}{2}}$ for the sequence $\tilde{u}_{n}$. Without loss of generality, suppose that $x_{0}=(1,0)$ is a concentration point of $\tilde{u}_{n}$. Then $\tilde{u}_{n}$ has at least two concentration points, $(0,0)$ and $(1,0)$. For $R>2$, consider $\tilde{u}_{n}$ on the ball $B_{R}^{1}=B_{R}\left(\left(-R+\frac{2}{3}, 0\right)\right)$. It is easy to see that

$$
\begin{aligned}
& \lim _{n \rightarrow \infty}\left(E\left(\tilde{u}_{n}, B_{R}^{1}\right)-E\left(\tilde{u}, B_{R}^{1}\right)\right) \\
\leq & \lim _{R_{0} \rightarrow \infty} \lim _{n \rightarrow \infty}\left(E\left(\tilde{u}_{n}, B_{R_{0}}\right)-E\left(\tilde{u}, B_{R_{0}}\right)\right)-\lim _{\delta \rightarrow 0} \lim _{n \rightarrow \infty} E\left(\tilde{u}_{n}, B_{\delta}\left(x_{0}\right)\right) \\
\leq & \lim _{\delta \rightarrow 0} \lim _{n \rightarrow \infty} E\left(u_{n}, B_{\delta}\right)-\epsilon_{N} \\
< & k \epsilon_{N} .
\end{aligned}
$$

By the induction assumption we can construct the bubble tree of $\tilde{u}_{n}$ on the ball $B_{R}^{1}$. Similarly, we can obtain the bubble tree of $\tilde{u}_{n}$ on the ball $B_{R}^{2}=B_{R}\left(\left(R+\frac{2}{3}, 0\right)\right)$. It is easy to see that for any $x$, if $R$ is large enough, then $x \in B_{R}^{1} \cup B_{R}^{2}$. So we obtain the bubble tree of $\tilde{u}_{n}$.

Case 2. If there is no concentration point on $B_{4} \backslash B_{\frac{1}{2}}$ for the sequence $\tilde{u}_{n}$, then we know that $\tilde{u}$ is a nonconstant harmonic map and the origin 0 is a concentration point for the sequence $\tilde{u}_{n}$. So there holds

$$
\begin{aligned}
\lim _{R \rightarrow \infty} \lim _{n \rightarrow \infty}\left(E\left(\tilde{u}_{n}, B_{R}\right)-E(\tilde{u})\right) & \leq \lim _{R \rightarrow \infty} \lim _{n \rightarrow \infty} E\left(\tilde{u}_{n}, B_{R}\right)-\epsilon_{N} \\
& =\lim _{\delta \rightarrow 0} \lim _{n \rightarrow \infty} E\left(u_{n}, B_{\delta}\right)-\epsilon_{N} \\
& \leq \lim _{n \rightarrow \infty}\left(E\left(u_{n}\right)-E(u)\right)-\epsilon_{N} \\
& <k \epsilon_{N} .
\end{aligned}
$$

We can also get the bubble tree $\tilde{u}_{n}$ on $R^{2}$ by the induction assumption.

Now assume that all the bubbles of $\tilde{u}_{n}$ are produced by $\tilde{u}_{n}\left(y_{n}^{i}+s_{n}^{i} y\right)$. By the selection of $t_{n}$, for any $x_{n}, r_{n}$ with $\frac{\left|x_{n}\right|+r_{n}}{t_{n}} \rightarrow \infty$, one can see that $u_{n}\left(x_{n}+r_{n} x\right) \rightarrow 0$ weakly in $W_{L o c}^{1,2}\left(R^{2}, N\right)$, i.e. any bubble of $u_{n}$ is a bubble of $\tilde{u}_{n}$ or produced by $\tilde{u}_{n}$ itself. Set $x_{n}^{i}=t_{n} y_{n}^{i}, r_{n}^{i}=t_{n} s_{n}^{i}$. If $\tilde{u}$ is constant, we get that all the bubbles of $u_{n}$ are produced by $u_{n}\left(x_{n}^{i}+r_{n}^{i} y\right)$. If $\tilde{u}$ is nonconstant, $\tilde{u}_{n}=u_{n}\left(t_{n} x\right)$ produces a new bubble $\tilde{u}$. So we prove the theorem. 
Remark. Consider $\triangle u_{n}=f_{n}=n^{2} f(n x)$ on $B_{1}$ where $f \in C_{0}^{\infty}\left(B_{1}\right) \geq 0$. It is easy to see that this theorem is false if we require only that $\tau\left(u_{n}\right)$ are bounded in $L^{1}$.

\section{REFERENCES}

[1] Weiyue Ding and Gang Tian, Energy identity for a class of approximate harmonic maps from surfaces, Comm. Anal. Geom. 3 (1995), no. 3-4, 543-554. MR1371209 (97e:58055)

[2] Jiayu Li and Xiangrong Zhu, Small energy compactness for approximate harmomic mappings, Commun. Contemp. Math. 13 (2011), no. 5, 741-763, DOI 10.1142/S0219199711004427. $\operatorname{MR} 2847227$

[3] J. Li and X. Zhu, Energy identity for the maps from a surface with tension field bounded in $L^{p}$. Preprint.

[4] Fanghua Lin and Changyou Wang, Energy identity of harmonic map flows from surfaces at finite singular time, Calc. Var. Partial Differential Equations 6 (1998), no. 4, 369-380, DOI 10.1007/s005260050095. MR1624304 (99k:58047)

[5] Thomas H. Parker, Bubble tree convergence for harmonic maps, J. Differential Geom. 44 (1996), no. 3, 595-633. MR.1431008 (98k:58069)

[6] Jie Qing, On singularities of the heat flow for harmonic maps from surfaces into spheres, Comm. Anal. Geom. 3 (1995), no. 1-2, 297-315. MR1362654 (97c:58154)

[7] Jie Qing and Gang Tian, Bubbling of the heat flows for harmonic maps from surfaces, Comm. Pure Appl. Math. 50 (1997), no. 4, 295-310, DOI 10.1002/(SICI)10970312(199704)50:4〈295::AID-CPA1〉3.0.CO;2-5. MR 1438148 (98k:58070)

[8] Peter Topping, Repulsion and quantization in almost-harmonic maps, and asymptotics of the harmonic map flow, Ann. of Math. (2) 159 (2004), no. 2, 465-534, DOI 10.4007/ annals.2004.159.465. MR2081434(2005g:58029)

Department of Mathematics, Zhejiang Normal University, Jinhua, Zhejiang 321004 , People's Republic of China

E-mail address: zxr701197@hotmail.com 